\title{
Electronic Structure and Magnetic Anisotropy in Lanthanoid Single-Ion Magnets with C3 Symmetry: The Ln(trenovan) Series
}

Questa è la Versione finale Accepted manuscript della seguente pubblicazione:

Original Citation:

Electronic Structure and Magnetic Anisotropy in Lanthanoid Single-lon Magnets with C3 Symmetry: The Ln(trenovan)

Series / Lucaccini, Eva; Baldovã॰, Josã@ J.; Chelazzi, Laura; Barra, Anne-laure; Grepioni, Fabrizia; Costes, Jean-pierre;

Sorace, Lorenzo. - In: INORGANIC CHEMISTRY. - ISSN 0020-1669. - STAMPA. - 56(2017), pp. 4728-4738.

[10.1021/acs.inorgchem.7b00413]

Availability:

This version is available at: $2158 / 1101098$ since: $2019-12-12 T 15: 01: 45 Z$

Published version:

DOI: $10.1021 /$ acs.inorgchem.7b00413

Terms of use:

Open Access

La pubblicazione è resa disponibile sotto le norme e i termini della licenza di deposito, secondo quanto stabilito dalla

Policy per l'accesso aperto dell'Università degli Studi di Firenze (https://www.sba.unifi.it/upload/policy-oa-2016-1.pdf)

Publisher copyright claim:

(Article begins on next page) 


\title{
Electronic structure and magnetic anisotropy in lanthanoid single-ion mag- nets with $C_{3}$ symmetry: the $\operatorname{Ln}($ trenovan) series
}

\author{
Eva Lucaccini, ${ }^{[a] \neq}$ José J. Baldoví, ${ }^{[b] \ddagger^{*}}$ Laura Chelazzi, ${ }^{[c]+}$ Anne-Laure Barra, ${ }^{[\mathrm{d}]}$ Fabrizia Grepioni, $\left.{ }^{[c]}\right]^{+}$Jean-Pierre Costes, ${ }^{[\mathrm{e}]}$ \\ Lorenzo Sorace ${ }^{[a]^{*}}$ \\ [a] Department of Chemistry “U.Schiff” and UdR INSTM, Università degli Studi di Firenze, Via della Lastruccia 3-13, 50019 Sesto Fiorentino (FI), \\ Italy. \\ [b] Nano-Bio Spectroscopy Group and European Theoretical Spectroscopy Facility (ETSF), Universidad del País Vasco CFM CSIC-UPV/EHU- \\ MPC \& DIPC, Av. Tolosa 72, 20018 San Sebastián, Spain \\ [c] Department of Chemistry “G. Ciamician”, Università degli Studi di Bologna, Via Selmi 2, 40196 Bologna, Italy. \\ [d] LNCMI-CNRS, 25 rue des Martyrs BP 166, 38042 Grenoble Cedex 9, France. \\ [e] Laboratoire de Chimie de Coordination du CNRS, 205, route de Narbonne, BP 44099, 31077 Toulouse Cedex 4, France.
}

KEYWORDS: Single-molecule magnet, lanthanides, crystal field, CONDON

Supporting Information Placeholder

ABSTRACT: We report the syntheses and the magnetic characterization of a new series of lanthanide complexes, in which the $\mathrm{Ce}, \mathrm{Nd}, \mathrm{Gd}, \mathrm{Dy}, \mathrm{Er}$ and $\mathrm{Yb}$ derivatives show single-molecule magnet behavior. These complexes, named $\mathrm{Ln}$ (trenovan), where $\mathrm{H}_{3}$ trenovan is tris $(((3-$ methoxysalicylidene)amino)ethyl)amine), exhibit trigonal symmetry and the $\operatorname{Ln}$ (III) ion is hepta-coordinated. Their molecular structure is then very similar to that of the previously reported $\mathrm{Ln}$ (trensal) series, where $\mathrm{H}_{3}$ trensal is 2,2',2"Tris-(salicylideneimino)triethylamine. This prompted us to use the spectroscopic and magnetic properties of the $\mathrm{Ln}$ (trensal) family $(\mathrm{Ln}=\mathrm{Nd}, \mathrm{Tb}, \mathrm{Dy}, \mathrm{Ho}, \mathrm{Er}$ and $\mathrm{Tm})$ to obtain a set of CFPs to be used as starting point to determine the electronic structures and magnetic anisotropy of the analogous $\mathrm{Ln}$ (trenovan) complexes using the CONDON computational package. The obtained results were then used to discuss the EPR and AC susceptibility results. As a whole, the obtained results indicate that this type of complexes does not behave as 'real' Single Molecule Magnets, due to a charge distribution of the ligand around the lanthanoid which results in highly mixed ground states in terms of $M_{j}$ composition of the states. The crucial parameter in determining the slow relaxation of the magnetization is then rather the number of unpaired electrons, only Kramers' ions showing infield slow relaxation than the shape of charge distribution for different $\operatorname{Ln}(\mathrm{III})$.

\section{INTRODUCTION}

The discovery of slow relaxation of magnetization on terbium-phthalocyaninate by Ishikawa and co-workers ${ }^{1}$ was a breakthrough in molecular magnetism and, since then, mononuclear lanthanide single-molecule magnets (SMMs) have been coming out on top. ${ }^{2}$ The interesting magnetic behavior of lanthanide ions arises from a strong magnetic anisotropy and a large total angular momentum $\mathrm{J},{ }^{3}$ which are the fundamental keys to potentially observe single-molecule magnetism. ${ }^{4,5}$ Indeed, these systems have been proposed as potential candidates for applications in molecular spintronics, high density storage and quantum information processing. ${ }^{6,7}$ However, any future technological achievement is subordinate to a deepened understanding of the behavior of these molecular nanomagnets, starting from the clarifications of the different mechanisms involved in the relaxation of magnetization. ${ }^{8}$

From an empirical point of view, it is fundamental to use a combined approach of spectroscopic and magnetic characterization, in order to have a detailed picture of the electronic structure in these complexes., ${ }^{9,10}$ In this context, $\mathrm{Ln}$ (trensal) (where $\mathrm{H}_{3}$ trensal, sketched in Scheme 1, is 2,2',2"'Tris-(salicylideneimino)triethylamine) series has been recently shown to be a good benchmark, since its luminescence investigation has been known for a few years. ${ }^{11}$ The phenomenological determination of the symmetry-allowed crystal-field parameters (CFPs), derived from the luminescence spectra, allowed to estimate the magnetic properties of the family which were compared with experimental results. In some cases, small discrepancies were found, ${ }^{12}$ especially for those com- 
pounds whose lowest energy levels were not spectroscopically observed. This emphasizes the need to take into account also the magnetic and EPR characterization for an accurate description of the ground-multiplet crystalfield splitting, to which these techniques are more sensitive.

From a theoretical point of view, the CFPs can be calculated using the molecular crystal structures using different approaches. These allow the prediction of spectroscopic and magnetic properties and the rational design of new derivatives exhibiting SMM behavior.5 The simplest and earliest developed procedure is based on the point charge electrostatic model (PCEM) ${ }^{13}$ which was later improved by several semi-empirical models..$^{14-17}$ These models, while useful for computationally inexpensive predictions and for determining an initial set of CFPs, also need parametrization to take into account covalency, in order to provide a more realistic description of the system. A computationally more expensive approach is based on the calculation of the energy levels using post Hartree-Fock multi-configurational calculations, the socalled $a b$ initio methods. ${ }^{18}$ In general, this methodology has been widely used for the theoretical characterization of SMMs, as first principles methods are expected to provide results closer to reality than the more intuitive additive electrostatic methods. Nevertheless, deviations between theoretical and experimental observables have recently been accumulating, ${ }^{19-22}$ revealing that modeling the properties of $f$-element complexes from first principles still remains an open problem. This is a particularly compelling issue when comparison between structurally related systems with minor chemical differences are investigated, since the resulting differences in the energy patterns can easily be smaller than the uncertainty provided by the calculations. In such cases the analysis of the magnetic behavior of the complete Ln series may also provide some precious information about the observed trend along the series.

For these reasons we decided to investigate the static and dynamic properties of a new family of lanthanide

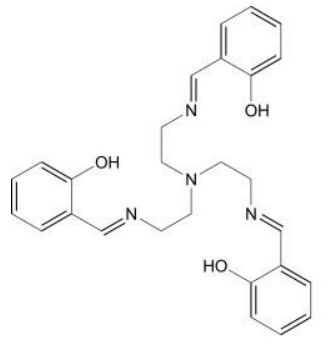

$\mathrm{H}_{3}$ trensal

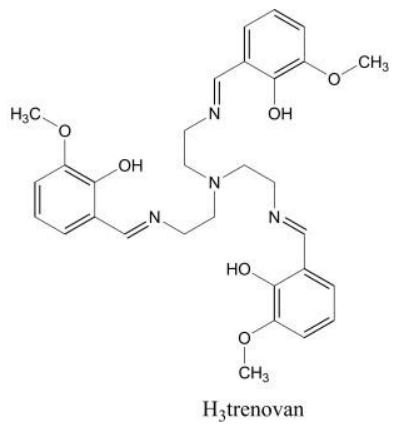

complexes, $\mathrm{Ln}$ (trenovan) (where $\mathrm{H}_{3}$ trenovan, sketched in Scheme 1, is tris (( (3-methoxysaliclidene)amino)ethyl)amine) and $\mathrm{Ln}=\mathrm{Ce}, \mathrm{Pr}, \mathrm{Nd}, \mathrm{Sm}, \mathrm{Eu}, \mathrm{Gd}, \mathrm{Tb}, \mathrm{Dy}$, $\mathrm{Ho}, \mathrm{Er}, \mathrm{Tm}$ and $\mathrm{Yb}$ ) which only differs in a methoxysubstituted position on the ligand, from the above mentioned $\operatorname{Ln}$ (trensal) family. For the latter series we report here the static magnetic characterization which is used, in combination with the previously reported luminescence data, ${ }^{11}$ to obtain an improved description of the electronic structure of this series.

This was then used as a starting point for the characterization of $\operatorname{Ln}($ trenovan) complexes. Analogously to $\operatorname{Ln}$ (trensal), this new series features crystallographically imposed trigonal symmetry (Figure 1). However, due to the impossibility to obtain crystalline samples and their corresponding spectroscopic and/or single-crystal measurements, we had to follow a different approach. We then resorted to a phenomenological approach, by using the CONDON computational package. ${ }^{23,24}$ This was first used to extract a new set of CFPs for each $\operatorname{Ln}$ (trensal) derivative prepared, $\mathrm{Ln}=\mathrm{Nd}, \mathrm{Tb}, \mathrm{Dy}, \mathrm{Ho}$, Er and Tm, by simultaneously fitting all the available spectroscopic and magnetic data. Subsequently, we used these improved sets of CFPs obtained for $\mathrm{Ln}$ (trensal) as initial guesses for the determination of the CFPs of $\operatorname{Ln}($ trenovan). Such CFPs are obtained by performing a fit of magnetic susceptibility taking into account the Zeeman splitting of the ground doublet via the $g$-factors experimentally determined by EPR.

\section{EXPERIMENTAL SECTION}

\section{Ln(trenovan) family}

Synthesis. All starting materials were purchased from Aldrich and used without further purification. The $\mathrm{H}_{3}$ trenovan ligand, tris $(((3-$ methoxysalicylidene $)$ amino)ethyl)amine, resulting from reaction of tris(2aminoethyl)amine with orthovanillin was obtained as previously described. ${ }^{25-27}$ The different complexes were prepared according to two different experimental pathways. For derivatives from $\mathrm{Ce}$ to $\mathrm{Sm}$, the previously published two-step process ${ }^{26}$ was retained while a one-step reaction was used for complexes going from $\mathrm{Eu}$ to $\mathrm{Yb}$. An example of each preparation process will be given for $\mathrm{Ce}$ and Eu complexes; yield, elemental, infrared data will be reported for the other complexes. Elemental analyses were carried out at the Laboratoire de Chimie de Coordination Microanalytical Laboratory in Toulouse, France, 
for C, H, and N. IR spectra were recorded with a PerkinElmer Spectrum 100FTIR using the ATR mode (see also Supplementary Material).

$\mathbf{C e}($ trenovan $)\left(\mathbf{H}_{2} \mathrm{O}\right)$. Addition of $\mathrm{Ce}\left(\mathrm{NO}_{3}\right)_{3} \cdot 5 \mathrm{H}_{2} \mathrm{O}(0.21$ $\left.\mathrm{g}, 5 \times 10^{-4} \mathrm{~mol}\right)$ to $\mathrm{H}_{3} \mathrm{~L}\left(0.275 \mathrm{~g}, 5 \times 10^{-4} \mathrm{~mol}\right)$ in methanol $(10 \mathrm{~mL})$ gave a yellow precipitate which was filtered off $30 \mathrm{~min}$ later. This precipitate corresponds to the $\mathrm{H}_{3} \mathrm{LCe}\left(\mathrm{NO}_{3}\right)_{3}$ entity. The precipitate was suspended and stirred at room temperature in methanol $(10 \mathrm{~mL})$. Addition of triethylamine $\left(0.3 \mathrm{~g}, 3.0 \times 10^{-3} \mathrm{~mol}\right)$ yielded a new pale yellow precipitate which was filtered off and washed with methanol and then diethyl ether. Yield: $0.13 \mathrm{~g}$ (36 \%). Anal. Calcd for $\mathrm{C}_{30} \mathrm{H}_{35} \mathrm{CeN}_{4} \mathrm{O}_{7}\left(703.75 \mathrm{~g} \mathrm{~mol}^{-1}\right)$ : C, 51.20; H, 5.01; N, 7.96. Found: C, 50.58; H, 4.75; N, 7.72. IR (ATR, $\mathrm{cm}^{-1}$ ): $3405 \mathrm{br}, 1621 \mathrm{~s}, 1593 \mathrm{~m}, 1545 \mathrm{w}$, $1466 \mathrm{~m}, 1439 \mathrm{~s}, 1403 \mathrm{~m}, 1332 \mathrm{~m}, 1319 \mathrm{~m}, 1240 \mathrm{~m}, 1215 \mathrm{~s}$, $1191 \mathrm{w}, 1164 \mathrm{w}, 1082 \mathrm{~m}, 1066 \mathrm{w}, 1033 \mathrm{~m}, 966 \mathrm{w}, 915 \mathrm{w}$, $854 \mathrm{~m}, 738 \mathrm{~m}, 622 \mathrm{w}$.

$\mathrm{Eu}$ (trenovan). Triethylamine $\left(0.3 \mathrm{~g}, 3.0 \times 10^{-3} \mathrm{~mol}\right)$ was added to a mixture of $\mathrm{Eu}\left(\mathrm{NO}_{3}\right)_{3} \cdot 5 \mathrm{H}_{2} \mathrm{O}(0.84 \mathrm{~g}, 2.0 \mathrm{x}$ $\left.10^{-3} \mathrm{~mol}\right)$ and $\mathrm{H}_{3} \mathrm{~L}\left(0.275 \mathrm{~g}, 2.0 \times 10^{-3} \mathrm{~mol}\right)$ in dimethylformamide $(10 \mathrm{~mL})$. The resulting mixture was stirred and heated for $20 \mathrm{~min}$, yielding a yellow precipitate, which was filtered off after cooling, washed with cold $\mathrm{dmf}$ and then diethyl ether. Yield: $0.25 \mathrm{~g}(71 \%)$. Anal. Calcd for $\mathrm{C}_{30} \mathrm{H}_{33} \mathrm{EuN}_{4} \mathrm{O}_{6}\left(697.58\right.$ gmol $\left.^{-1}\right)$ : C, 51.65; $\mathrm{H}, 4.77 ; \mathrm{N}$, 8.03. Found: C, 51.33; H, 4.67; N, 7.92. IR (ATR, $\left.\mathrm{cm}^{-1}\right)$ : $1623 \mathrm{~s}, 1594 \mathrm{~m}, 1546 \mathrm{w}, 1467 \mathrm{~m}, 1441 \mathrm{~s}, 1404 \mathrm{~m}, 1332 \mathrm{~m}$, $1323 \mathrm{~m}, 1240 \mathrm{~m}, 1217 \mathrm{~s}, 1192 \mathrm{w}, 1165 \mathrm{w}, 1083 \mathrm{~m}, 1065 \mathrm{w}$, $1034 \mathrm{~m}, 966 \mathrm{w}, 917 \mathrm{w}, 857 \mathrm{~m}, 748 \mathrm{~m}, 739 \mathrm{~m}, 623 \mathrm{w}$.

$\mathrm{X}$-ray powder diffraction (XRPD) experiments. For structure solution and refinement data polycrystalline samples of $\operatorname{Pr}($ trenovan), Gd(trenovan) and $\mathrm{Tm}$ (trenovan) were sealed in a $0.5 \mathrm{~mm}$ capillary, and an $\mathrm{X}$-ray powder diffractogram in the $2 \theta$ range $3-60^{\circ}$ (step size $0.01^{\circ}$, time/step $2 \mathrm{~s}, V=40 \mathrm{kV}, I=40 \mathrm{mAA}$ ) was collected on a D8 Bruker diffractometer equipped with a primary $\mathrm{Ge}$ monochromator for $\mathrm{Cu} \mathrm{K}_{\mathrm{al}}$ and a Sol-X solid state detector in Debye-Scherrer geometry.

Structure determination from XRPD data. For $\operatorname{Pr}$ (trenovan), $\mathrm{Gd}($ trenovan) and $\mathrm{Tm}$ (trenovan), a volume of about $1427 \AA^{3}, 1426 \AA^{3}$ and $1417 \AA^{3}$ was found respectively with the algorithm DICVOL. ${ }^{28}$ The asymmetric unit contains one third of the molecule. Space group determination with Highscore plus resulted for the three structure in space group P-3, with $\mathrm{Z}=6$. The structure of $\operatorname{Tm}$ (trenovan) was solved by simulated annealing that runs with structure fragments. Some weak peaks of the diffractogram turned out to belong to a different unassigned phase. They have then been excluded from the structure solution procedure. The final agreement between experimental and calculated diffractogram is however satisfactory indicating a small contribution of the spurious phase (see Figure S1)

Simulated annealing was performed with EXPO2013, ${ }^{29}$ using $1 / 3$ of the molecule plus the nitrogen and the ion Tm constrained to lie along the $C_{3}$ axis. Ten runs for simulated annealing trial were set, and a cooling rate (defined as the ratio $\mathrm{Tn} / \mathrm{Tn}-1)$ of 0.95 was used. Best solutions were chosen for Rietveld refinements, which were performed with the software TOPAS. ${ }^{30}$ A shifted Chebyshev function with 16 parameters and a Pseudo-Voigt function were used to fit background and peak shape, respectively. Soft constraints were applied for all bond distances and

Table 1: Crystal structure data for $\operatorname{Pr}($ trenovan), Gd(trenovan) and $\mathrm{Tm}($ trenovan)

\section{$\operatorname{Pr}(\operatorname{trenovan})$ \\ Gd(trenovan)}

$\begin{array}{cc}\mathrm{C}_{30} \mathrm{H}_{33} \mathrm{~N}_{4} \mathrm{O}_{6} \mathrm{Gd}_{1} & \mathrm{C}_{30} \mathrm{H}_{33} \mathrm{~N}_{4} \mathrm{O}_{6} \mathrm{Tm}_{1} \\ 702.8 & 714.5 \\ 293 & 293 \\ 1.54056 & 1.54056 \\ \text { trigonal } & \text { trigonal } \\ \boldsymbol{P}-3 & \boldsymbol{P}-3 \\ 14.240(1) & 14.001(1) \\ 14.240(1) & 14.001(1) \\ 8.121(1) & 8.347(1) \\ 1426.25(1) & 1417.11(1) \\ 6 & 6 \\ 5.6 & 9.7 \\ 4.3 & 7.9\end{array}$


angles of the Tm(trenovan) molecule. An overall thermal parameter was adopted for all atoms of the structure. All the hydrogen atoms were fixed in calculated positions. Refinement converged with $\chi^{2}=1.28$ and Rwp $=9.68$ (see Table 1). The structure of $\operatorname{Pr}($ trenovan) and $\mathrm{Gd}$ (trenovan) were solved starting from the solution obtained for $\operatorname{Tm}$ (trenovan) considering the three structures isomorphic. The unit cell parameters, atomic coordinates and peak shape were refined (see Figure 1 for the molecular structure).

Determination of unit cell parameters (Pawley refinement). Powder diffraction data for 12 different $\operatorname{Ln}$ (trenovan) structures were analyzed with the software Highscore plus. 15 peaks were chosen in the $2 \theta$ range 3$40^{\circ}$, and unit cell parameters were found thanks to the algorithm DICVOL. Pawley refinement was performed for all the 12 unit cell parameters and results were shown in Table (S1).

\section{$\operatorname{Ln}($ trensal) family}

Synthesis and XRPD measurements. The Ln(trensal) complexes ( $\mathrm{Ln}=\mathrm{Nd}, \mathrm{Tb}, \mathrm{Dy}, \mathrm{Ho}, \mathrm{Er}, \mathrm{Tm})$, where $\mathrm{H}_{3}$ trensal $=2,2^{\prime}, 2^{\prime \prime}$-Tris-(salicylideneimino)trimethylamine, were synthetized as previously described. ${ }^{31} \mathrm{X}$-ray powder diffraction patterns of all compounds were measured with a Bruker D8 Advance powder diffractometer equipped with a Cu source $\left(\mathrm{K}_{a}, \lambda=1.54 \AA\right)$.

\section{Magnetic characterization and electronic struc- ture}

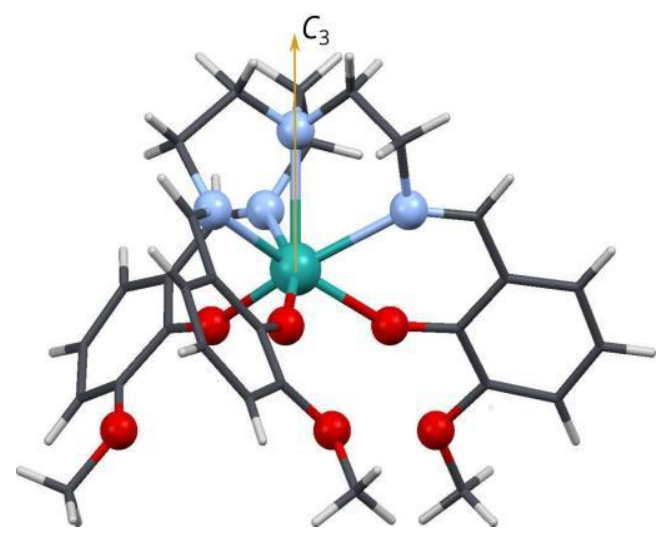

Magnetic Measurements. For both $\operatorname{Ln}($ trenovan) and Ln(trensal) compounds DC magnetic measurements were performed by using a Quantum Design MPMS SQUID magnetometer on powders pressed in a pellet to avoid field induced orientation of the crystallites. For $\mathrm{Ln}$ (trenovan) series, raw data were rescaled by taking into account the presence of residual $\mathrm{dmf}$ lattice solvent, as estimated by TGA (see Table S2). Diamagnetic corrections were calculated using Pascal's constants ${ }^{32}$ and are available in the Supplementary Material.

The concentration of Dy(trenovan) and Gd(trenovan) in Y(III) diluted sample was estimated by the scaling factor necessary to superimpose the magnetization curve versus field of pure and diluted compound at low temperature. AC susceptibility was measured using Quantum Design PPMS in AC mode for the frequency range 10 to $10^{4} \mathrm{~Hz}$. The Quantum Design MPMS SQUID magnetometer was used for low frequencies $\left(0.02\right.$ to $\left.10^{3} \mathrm{~Hz}\right)$.

Computational details. The electronic structures of $\mathrm{Ln}$ (trensal) and $\mathrm{Ln}($ trenovan$)$ complexes $(\mathrm{Ln}=\mathrm{Nd}, \mathrm{Tb}$, $\mathrm{Dy}, \mathrm{Ho}, \mathrm{Er}$ and $\mathrm{Tm}$ ) were determined using the latest version of CONCORD code, which belongs to the CONDON package. This program permits to describe the spectroscopic and magnetic properties in $d$ and $f$ systems with high local symmetry using the full basis of microstates. In the first family, we have used as an input the parameters of the Ligand-Field Hamiltonian determined by Flanagan $e t$ al. (electron repulsion parameters: $F^{k}, \mathrm{k}=$ $2,4,6)$, spin-orbit coupling constant: $\xi_{\text {so }}$ and the nonnegligible crystal field parameters for $C_{3}$ symmetry: $B_{0}^{2}$,

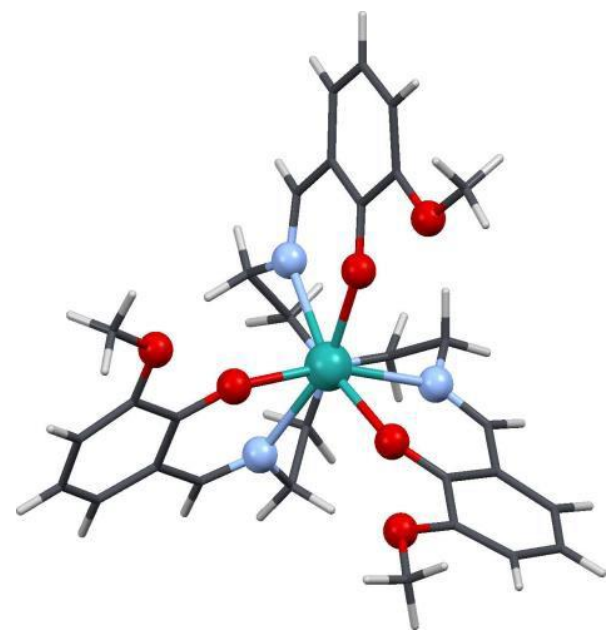

Figure 1: Left panel: view along the crystallographic axis $a$ of a molecule of $\operatorname{Pr}($ trenovan), as representative of the whole series: the Ln ion (green) is coordinated to 3 oxygens (red) and 4 nitrogens (light blues). Right panel: view along the $c$ axis. 
$B_{0}^{4} B_{0}^{6} B_{3}^{4} B_{3}^{6}$ and $\left.B_{6}^{6}\right)$. In arder to model the properties of the series $\operatorname{Ln}$ ( trensal), all the available luminescence spectra as well as the magnetic susceptibility data have been simultaneously fitted. In the case of Er(trensal) we have also taken advantage of the magnetic properties in the easy axis direction reparted by Pedersen et $a l .{ }^{19}$ ln the CONDON package, the goodness of the fit (SQX) is defined as follows:

$$
S Q X=\frac{{\sqrt{\sum_{i=0}^{\sum^{n}} q \cdot\left(1-\chi_{\text {theo }}\right)^{2}}}_{n}^{\chi_{\text {exp }}}}{n}
$$

where $\chi_{\text {theo }}$ and $\chi_{\text {exp }}$ are the calculated and measured magnetic susceptibility respectively, $\sigma_{\mathrm{i}}$ is a weighting factor, which as standard is I, and $n$ the number of points included in the fit. In the $\mathrm{Ln}$ ( trensal) family, where spectroscopic information has been included, the same definition of

SQX works, but in this case replacing $\chi$ by E. In the cases in which experimental energy levels from the ground multiplet were available (Er and Tm), such levels have been weighted using $\sigma=$ O.I, giving priarity to them in the fitting procedure, in arder to obtain a better description of the magnetic properties

In the compounds of the family Ln(trenovan), for which spectroscopic and single-crystal magnetometry data could not be obtained we combined the fit of the magnetic susceptibility data with that of the experimental $\mathrm{g}$;; value determined by EPR, which describes the evolution of the ground doublet under an applied magnetic field. The fitting procedure started from the CFPs calculated for the analogous Ln( trensal) derivatives.

EPR spectroscopy. X-band ( $\boldsymbol{\nu} \sim 9.4 \mathrm{GHz}$ ) and W-Band ( $\nu \sim 94 \mathrm{GHz}$ ) EPR spectroscopy was performed using commercia! Bruker ES00 and E600 spectrometer in the Laboratory of Molecular Magnetism, Florence. Multifrequency high field- EPR spectra were recorded on a homemade instrument at LNCMI - Grenoble. ${ }^{33}$ All the samples were undiluted except Gd(trenovan) and Dy( trenovan), for which the isostructural complexes YGd(trenovan) and YDy(trenovan) were studied (5.6\% and $8.2 \%$ of $\mathrm{Gd}(\mathrm{III})$ and $\mathrm{Dy}(\mathrm{III})$ respectively). Simulation of the W-band EPR spectrum of Gd(trenovan) was achieved using the Matlab toolbox Easyspin. ${ }^{34}$

\section{RESULTSAND DISCUSSION}

Syntheses. The lighter Ln complexes are prepared according to the previously published two-step process ${ }^{26}$ in which the first step corresponds to complexation of $\mathrm{Ln}$ ions by the outer0 303 coordination site of the tripodal ligand, with a proton transfer from the phenol function to the imine function. In that complex the ligand is in a zwitterionic phenoxo-iminium form, which implies the presence of nitrato anions as counter-ions. The resulting complex is isolateci and deprotonated in the second step by addition of triethylamine. Deprotonation involves a change of coordination site from the outer0 303 site to the inner N4O3 site. This method presents the advantage to keep a 1/1 ligand /Ln ratio all along the process. Although it can be applied to the entire Ln series, we have developed a one-step complexation reaction in arder to increase the final yield of the complexes involving the heavier Ln ions.

Structures and symmetries. Upon recrystallization the consistency of $\mathrm{Ln}$ (trensal) complexes $(\mathrm{Ln}=\mathrm{Nd}, \mathrm{Tb}$, Dy, $\mathrm{Ho}, \mathrm{Er}, \mathrm{Tm}$ ) with reported ones were checked by XRPD (Supplementary Materia!, Figures S2). The same technique was used to check for the isostructurality of Ln(trenovan) complexes along the series. The determination of the unit cell on the basis of the powder X-ray pattern reveals that the complexes crystallize in the space group $P$ - 3 (Table 1), and that the cell parameters vary regularly along the series (see Figure $\mathrm{S} 3$ ): the $e$ axis increases linearly on increasing the atomic number, while $a(b)$ contracts. Since the rate of variation of unit cell dimension vs $\mathrm{Z}$ is essentially the same for the two directions, the unit cell volume decreases as expected along the series $^{35}$ (see Figure S3). The structure of Pr(trenovan), Gd(trenovan) and Tm(trenovan) were then determined starting from powder data. As expected on the basis of the structure of complexes with closely similar ligand, the Ln center is heptacoordinated by three equivalent imino nitrogen atoms, three equivalent phenoxo- oxygen atoms, and an axial aminic nitrogen. Furthermore, the Ln ion sits on a $\mathrm{C} 3$ axis, a point of relevance for the subsequent analysis of the magnetic data (see below). In this respect the coordination geometry around the lanthanide is strictly similar to that observed in the $\operatorname{Ln}$ (trensal) series 313640 and complexes of relateci ligands. ${ }^{19}{ }_{-} 4143$ There is however a relevant difference compared to $\mathrm{Ln}$ ( trensal) series: as a first point, the Ln-N(amine) distance is much shorter in trenovan series than in trensal one, ranging from $2.57 \AA$ 


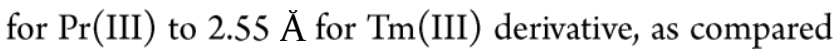

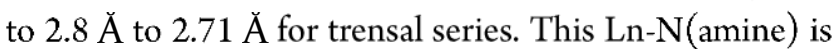
extremely short when compared to those observed in similar complexes, and suggests that the ligand field strength for $\operatorname{Ln}$ (trenovan) might be stronger than in $\mathrm{Ln}$ (trensal). It is worth noting that the differences in the Ln- $\mathrm{N}$ (imino) and in the Ln-O distances are much less important (see Table S3). Finally, we stress that the oxygen of the methoxy susbstituent are located at a non-

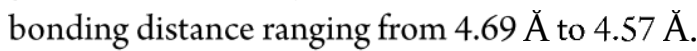

Since the lanthanide ions are lying on a $C_{3}$ site, the number of non-zero CFPs is reduced, and the crystal field splitting over the different multiplets can be described by means of the following CF Hamiltonian (expressed in the Wybourne formalism):

$$
\begin{aligned}
& \boldsymbol{H}_{\mathrm{CF}}=\underset{0}{B^{2} \boldsymbol{C}^{2}}+B_{0}^{4} \boldsymbol{C}^{4}{ }_{0}+\operatorname{Re}_{3}^{B^{4}}\left(\boldsymbol{C}^{4}-\boldsymbol{C}^{4}\right)+B_{3}^{6} \boldsymbol{C}_{00}^{6}
\end{aligned}
$$

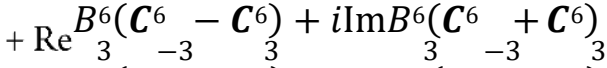

$$
\begin{aligned}
& \left.+\operatorname{Re}_{6}^{B^{6}\left(\boldsymbol{C}^{6}\right.}+\boldsymbol{C}_{6}^{6}\right)+{ }_{6} \operatorname{Im}_{6}^{6}\left(\boldsymbol{C}_{-6}^{6}-\boldsymbol{C}_{6}^{6}\right)
\end{aligned}
$$

Where $B_{q}^{k}$ are the Wybourne crystal field parameters, which are in general complex, with the exception of $q=0$. The real $\left(\operatorname{Re} B_{q}^{k}\right)$ and imaginary $\left(\operatorname{Im} B_{q}^{k}\right)$ parts are by definition real quantities. $\boldsymbol{C}_{q}^{k}$ are the Wybourne operators. $^{44,45}$

\section{Modeling the static magnetic properties.}

Compared to the previous investigations on $\mathrm{Ln}$ (trensal), we had to follow a different approach to analyze the magnetic and spectroscopic properties of $\operatorname{Ln}($ trenovan). Indeed, syntheses did not provide single crystal samples but only powders, so that it was not possible to perform a high resolution luminescence investigation or other single crystal characterization, like torque magnetometry, which were fundamental in elucidating the electronic structure of $\operatorname{Ln}$ (trensal) ${ }^{11,12} \mathrm{~A}$ possible path to gain information on the electronic structure, in cases like this one, would have involved performing theoretical calculations using the crystal structures as input. For that we could use $a b$ initio methods, but in this case we had to deal with a series of different problems: (1) The molecular structure were obtained from powder X-ray data which, despite their high quality, provide somehow larger uncertainties on atomic positions than single crystal ones; (2) it was also not possible to use a simplified, or optimized, molecular
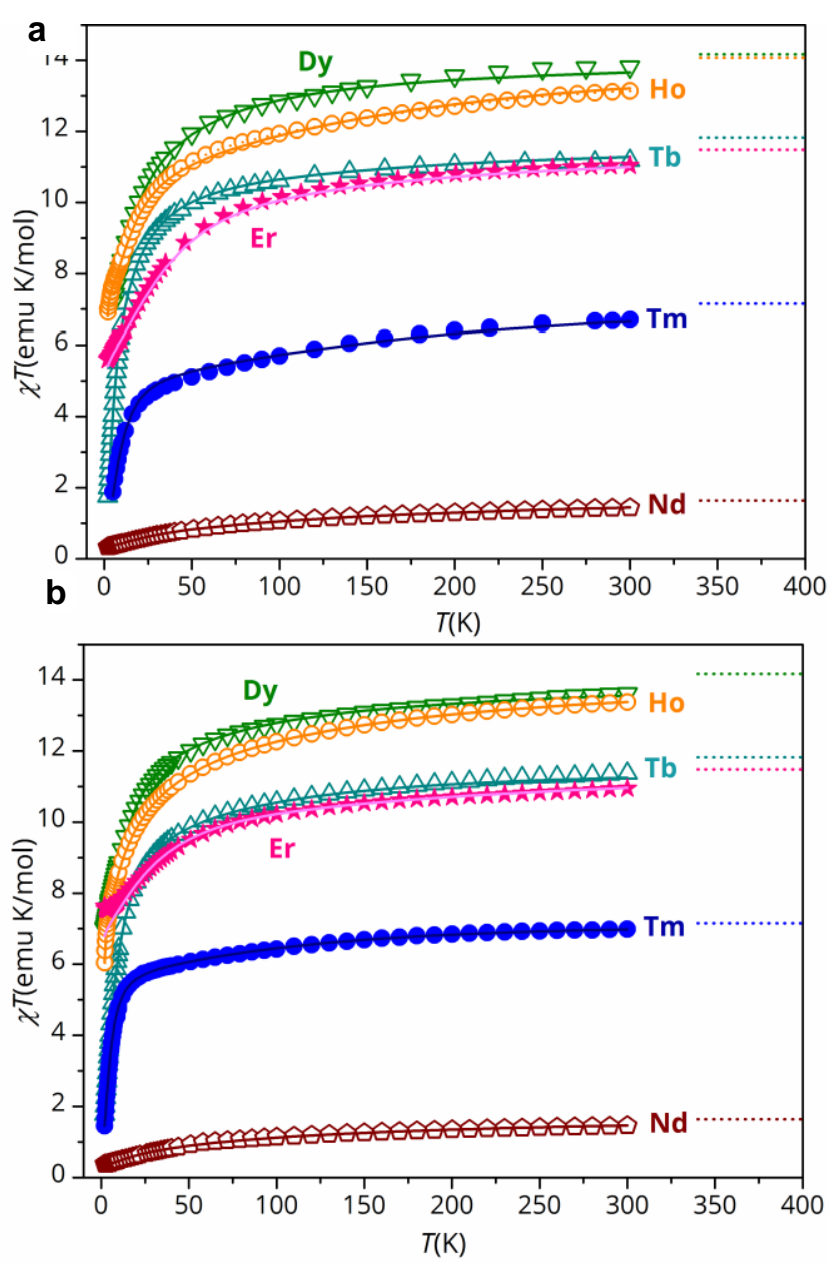

Figure 2: Experimental (symbols) and theoretical fit (solid lines) $\chi T$ product of the series $\operatorname{Ln}$ (trensal) (upper panel) and $\mathrm{Ln}$ (trenovan) (lower panel) from 2 to $300 \mathrm{~K}$ - The dotted lines represent the Curie constants of the corresponding free ions. Color scale: $\mathrm{Nd}$ (empty wine pentagons), $\mathrm{Tb}$ (cyan empty triangles), Dy (reversed green empty triangles), Ho (orange empty circles), Er (pink stars), Tm (full blue circles).

structure, since this would have amounted to assuming the same structure as $\operatorname{Ln}$ (trensal), making a comparison between the two series meaningless; (3) the calculations for the whole series was quite demanding and their validation on the basis of experimental studies ${ }^{9}$ would not have been so strong as for other complexes, due to the reasons outlined above. ${ }^{19,46}$ The latter point is even more relevant if one considers that the calculated CFPs by CASSCF in $\operatorname{Er}($ trensal $)$ and two related complexes by peripheral ligand functionalization were unable to reproduce the spectroscopic INS transitions nor the $g$-factors. ${ }^{19}$ On the other hand, the application of the semiempirical Radial Effective Charge (REC) model $^{17}$ using the SIMPRE computational 
package ${ }^{47,48}$ was hampered by the lanthanide being coordinated to three types of donar atoms (see Figure 1) and by SIMPRE considering only the ground multiplet. As this parametric model needs to introduce two parameters for each type of donor atom, the use of this approach would result in a total of six REC parameters leading to possible unphysical mathematical fits, whose extrapolation to predict the behavior of other compounds could not be reliable.

Thus, we have followed a purely phenomenological approach using the CONDON computational package. This procedure benefited of the large number of energy levels determined by spectroscopic means for $\operatorname{Ln}$ ( trensal) and of the high and coincident symmetry of the coordination environments in the two families. Firstly, we fitted all the energy levels determined by luminescence ${ }^{11}$ for the $\mathrm{Nd}$, $\mathrm{Tb}$, Dy, Ho and Tm derivatives of the $\operatorname{Ln}$ ( trensal) series, and the temperature dependences of their magnetic susceptibilities, which were determined in the course of this work. In the case of Er derivative, thanks to the more complete set of experimental information, we could simultaneously fit: (a) the $\mathrm{S} 1$ spectroscopic Kramers doublets from the different multiplets/ ${ }^{9}$ (b) the Zeeman splitting of the ground multiplet with field applied along the trigona! axis, using $g_{/ /}^{e f f}=11.8$ determined by EPRi (c) the magnetic susceptibility and magnetizations (2, 4 and $6 \mathrm{~K})$ measured along the $\mathrm{C}_{3}$ axis direction previously reported in literature ${ }^{19}$ (see Figure S4).

The temperature dependence of the powder magnetic susceptibility was then calculated using the obtained CFPs and compared with the experiment, obtaining an almost perfect agreement $(\mathrm{SQX}=0.46 \%)$. This case represents the best scenario for modeling the properties of $f$ element coordination complexes, i.e. having a combination of thermodynamic and spectroscopic information from different techniques ${ }^{50} 52$ and fitting all data by means of the full Hamiltonian. The $\chi T$ product of the six prepared derivatives of $\operatorname{Ln}$ (trensal) is plotted as a function of temperature in Figure 2a. The relative errors of the fitting procedure of the other derivatives vary between $2.21 \%$ in the case of $\mathrm{Tb}$ (trensal) to $0.54 \%$ in $\mathrm{Ho}$ (trensal) (see Table S4). The larger difference in the relative errar of the $\mathrm{Tb}$ derivative is related to the number of energy levels determined by luminescence spectroscopy (15 in the case of $\mathrm{Tb}$ and 59 in Ho), which forced us to keep free-ion parameters values fixed to avoid overparametrization. $\mathrm{Nev}$ ertheless, one can see that the overall reproduction of the experimental data of all the complexes of the series is very satisfactory. Indeed, the CFPs reported in Table S4 further allowed to calculate the $M$ vs $\mathrm{H}$ dependence through the homemade-software EVALUCF: ${ }^{53}$ the qualitative agreement between the experimental values and the calculated curves is quite good (Figure SS). The calculated energy levels and the ground state wavefunctions are reported in Table SS-S11: we note, in particular, that for Dy(trensal) the calculated energy of the first excited doublet is intermediate between the one obtained using only luminescence data ${ }^{54}$ ( $\mathrm{SO} \mathrm{cm}^{1}$ ) and that derived by torque magnetometry ${ }^{12}\left(19 \mathrm{~cm}^{1}\right)$.

Having obtained a reliable set of parameters for $\mathrm{Ln}$ (trensal), we used these ones as starting point for the rationalization of the magnetic behavior ofLn(trenovan). As a first qualitative observation, we note that the room temperature $\chi T$ values for both $\operatorname{Ln}$ (trensal) and for $\operatorname{Ln}($ trenovan) are near the expected values for the ions in absence of crystal field splitting (see Table S12) and quite dose to each other. The temperature dependence of the powder magnetic susceptibilities of $\operatorname{Ln}$ (trenovan) between 2 and $300 \mathrm{~K}$ (Figure 2b) were then fitted, including the Zeeman splitting of the ground Kramers doublet in a second step using the experimental $g / /$ value determined by EPR (see below). Subsequently, the CFPs were refined with a new fit of magnetic susceptibility using a few iterations more until SQX $<2 \%$. As can be observed the fitted $x T$ products (solid lines) are in excellent agreement with experimental data. All the obtained sets of CFPs as well as the ground multiplet energy levels and wavefunctions are available as supporting information (see Table S13-S20, Figure S6-S7).

The differences in CFPs obviously results in different wavefunction composition between $\mathrm{Ln}$ ( trensal) and $\mathrm{Ln}$ ( trenovan). If we focus on the ground state wavefunction we see that for Dy(trensal) the ground Kramers' doublet is described mainly by a mixture of $34 \% \mid \mp 7 / 2>$, $32 \% 1 \pm 5 / 2>$ and $23 \% 1 \bar{\mp}_{1 / 2}>$, whereas Dy(trenovan) is described by $69 \% 1 \bar{\mp}_{1 / 2>}$, followed by $17 \% 1 \bar{\mp}_{7 / 2>}$ and $12 \%$ of $\mid \pm 5 / 2>$. In the case of Er, the $\mid \pm 13 / 2>$ contribution to the ground state is enhanced from $77 \%$ in $\operatorname{Er}$ (trensal) to $92 \%$ in $\operatorname{Er}$ (trenovan), whereas in the case of the $\mathrm{Nd}$ derivatives, the dominant $M_{1}$ contribution is reduced from $82 \%$ of $\mid \pm 5 / 2>$ in $\mathrm{Nd}$ (trensal) to $72 \%$ of $\mid \pm 5 / 2>$ in $\mathrm{Nd}$ (trenovan). For the non-Kramers ions, all those investigated are characterized by a ground singlet: in $\mathrm{Tm}$ (trensal) this is dominated by a mixture of 
$40 \% \mid+6>$ and $40 \% \mid-6>$, with a contribution of $9 \% \mid+3>$ and $9 \% \mid-3>$, the first excited singlet being located at 20 $\mathrm{cm}^{-1}$; for $\operatorname{Tm}$ (trenovan) the ground state has $46 \%$ of $\mid+6>$ and $46 \%$ of $|-6\rangle$ contributions, with the excited state closer in energy $\left(11 \mathrm{~cm}^{-1}\right.$, see also Tables S11 and S20).

At this stage it is useful to make a comparison between the ligand field strength in the two series of complexes and its behavior along the series. This can be quantified as described in the paper of Riley and coworkers: ${ }^{11}$

$$
\left.\frac{N_{v}}{(4 \pi)^{1 / 2}}=\left\lceil\sum_{k=2,4,6}\left(\frac{1}{2 k+1}\right)\left(\|_{0}^{k^{2}}+2 \sum_{q=1}^{k} \mid \begin{array}{c}
B_{k} p \\
q
\end{array}\right)\right]\right]^{1 / 2}
$$

The results, reported both in Table S21 and graphically in Figure S8 confirms that, on increasing $Z$, the LF strength is decreasing for both series. Furthermore, the LF strength of the $\operatorname{Ln}$ (trenovan) series is slightly higher, on average, than for the $\operatorname{Ln}$ (trensal) one. While the first, counterintuitive behavior was attributed by Riley and coworkers to the contraction of the $f$ orbitals along the series, resulting in a reduced metal-ligand orbital overlap, the latter point can be traced back to the stronger interaction provided by the aminic nitrogen in $\mathrm{Ln}$ (trenovan) as a consequence of the shorter distance (see above). The clear anomalies observed for Ho and Tm trenovan derivatives further suggest that, despite the informed choice of starting values for CFP parameters and the agreement with the experimental properties, the best fit values obtained for these complexes might well be non- correct. This remarks once more that the use of the only magnetic data to obtain CFPs should be, in cases more fortunate than this one, avoided and complemented by spectroscopic information. ${ }^{50}$

Magnetic anisotropy. The magnetic anisotropy of the $\mathrm{Ln}$ (trenovan) series at low temperature was investigated by means of CW-EPR spectroscopy and compared to the corresponding analogues of $\operatorname{Ln}($ trensal $)$ series: this was

\begin{tabular}{c|ccccc} 
& $g^{\perp}(\exp )$ & $g^{\perp}($ calc $)$ & $g_{/ /}(\exp )$ & $g_{/ /}($calc $) \Delta$ & $g / \Sigma_{g}(\exp )$ \\
\hline $\mathrm{Ce}$ & $1.94(1)$ & - & Not visible & - & $<0$ \\
$\mathrm{Nd}$ & $0.68(1)$ & 1.60 & $2.62(1)$ & 2.24 & $0.588(8)$ \\
$\mathrm{YDy}$ & $8.7(2)$ & 10.36 & $1.63(1)$ & 1.88 & $-0.684(4)$ \\
$\mathrm{Er}$ & $2.37(1)$ & 2.38 & $13.54(1)$ & 14.32 & $0.702(1)$ \\
$\mathrm{Yb}$ & $2.643(6)$ & - & $4.92(1)$ & - & $0.301(1)$
\end{tabular}

Table 2: Experimental $g$-factor and calculated ones for the $\mathrm{Ln}$ (trenovan) series using the CFPs reported in Table S13. intended both as a feedback for the phenomenological approach described in the previous section and as a way to evidence any trend in the magnetic anisotropy between the two ligand families.

Kramers ions. For the sake of simplicity, we started our investigation by the Kramers' ions of the series, since for these systems resonance condition can be met at reasonably accessible frequency/field combination. The response of the ground doublet to the applied field can indeed be modeled by using an effective Zeeman Hamiltonian, for which the determined $g$ values embody the wavefunction composition. The crystallographically imposed trigonal symmetry of the $\operatorname{Ln}$ (III) center in the two series further assures a strict axial symmetry of the obtained effective $g$ factors, with either $g_{/ /}>g_{\perp}$ (easy axis anisotropy) or $g_{\perp}>g_{/ /}$(easy plane anisotropy). The Ln(trenovan) complexes exhibited both kinds of magnetic anisotropy: $\mathrm{Ce}, \mathrm{Dy}$ and Gd derivatives have an easy plane magnetic anisotropy, while $\mathrm{Nd}, \mathrm{Er}$ and $\mathrm{Yb}$ derivatives have an easy axis anisotropy (see Figure 3 and Table 2).

In the case of Ce derivative, the EPR investigation did not
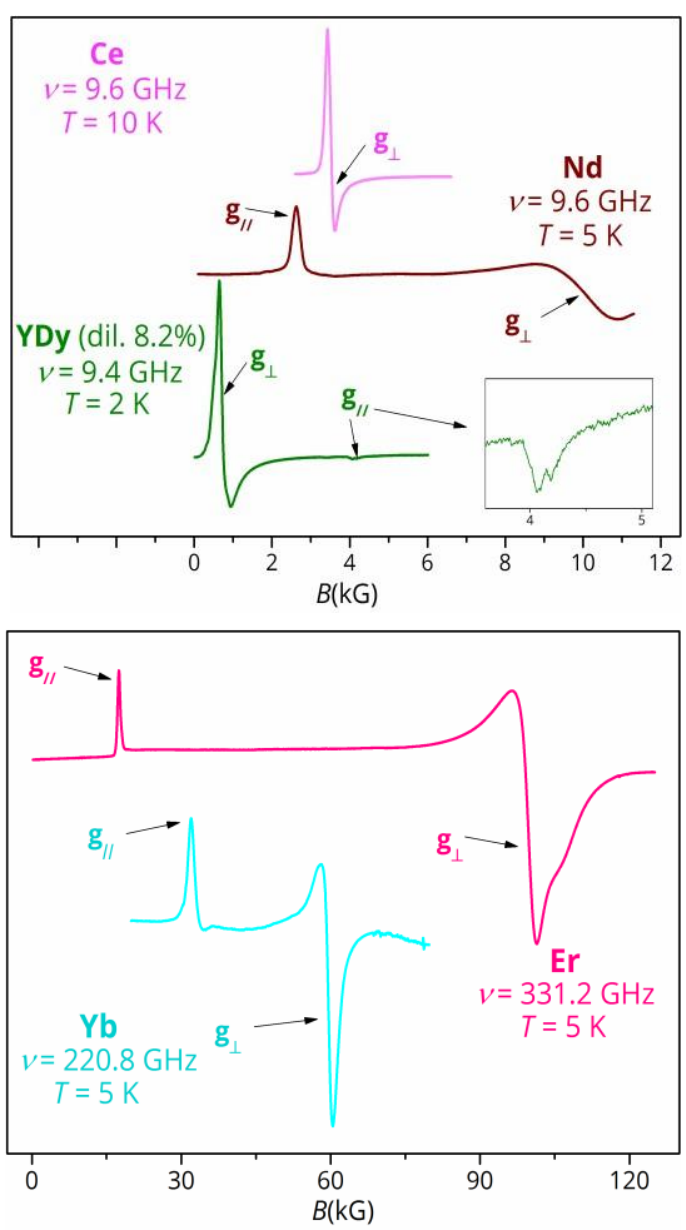

Figure 3: Upper panel: EPR spectra for Ce(trenovan), $\mathrm{Nd}$ (trenovan), YDy(trenovan) measured in $\mathrm{X}$ band. Lower panel: EPR sectra for $\operatorname{Er}($ trenovan) and $\mathrm{Yb}$ (trenovan) measured at LNMCI. 
detect any signal corresponding to a parallel transition. This can be easily rationalized by considering that $\mathrm{Ce}$ (III) has a ground multiplet with $J=5 / 2$, and that the trigonal site symmetry results in each doublet being either a mix of $M_{j}=\mid \pm 5 / 2>$ and $M_{j}=\mid \mp 1 / 2>$ or a pure $M_{j}=\mid \pm 3$ $/ 2>.^{8}$ For $g_{\perp} \neq 0$, the former situation should occur: further a simple analysis shows that, for $g_{/ /}$to be consistent with the observed $g_{\perp}$, its value should be around 0.40 , i.e. beyond the magnetic field limit of our X-band spectrometer. We stress here that, among the Kramers ions, the anisotropy of $\mathrm{Sm}$ (trenovan) could not be determined: indeed, the sample turned out to contain some Gd(III) impurity, its EPR spectrum featuring a striking similarity to the one of Gd(trenovan) diluted in the Y(III) diamagnetic matrix (see Figure S9). We attributed this behavior to the very low magnetic moment of $\mathrm{Sm}$ (trenovan) at low temperature, which then acts as an effective 'quasi-diamagnetic' matrix for the Gd(III) impurity dopants. No signal intrinsic to $\mathrm{Sm}$ (III) could be detected.

As a feedback for the phenomenological approach described in the previous section, the CFPs of Nd, Dy and Er were employed to get an estimation of their g-factor values by means of the homemade software EVALUCF. ${ }^{53}$ The comparison between the experimental and the calculated values is reported in Table 2, evidencing a good qualitative agreement.

The obtained results were further compared to those obtained on corresponding systems of $\operatorname{Ln}$ (trensal) for which CFP parameters have been obtained, i.e. $\mathrm{Nd}$ (trensal), Dy(trensal) and Er(trensal). The spectra are reported in Figure S10. In analogy with the $\operatorname{Ln}$ (trenovan) compounds, $\mathrm{Nd}$ (trensal) and $\mathrm{Er}$ (trensal) have easy axis magnetic anisotropy while Dy(trensal) is easy plane. ${ }^{54}$ Also in this case the effective $g$ factors calculated on the basis of the best fit CFP parameters turned out to be in good agreement with the experimental evidences (see

\begin{tabular}{c|ccccc} 
& $g^{\perp}(\exp )$ & $g^{\perp}($ calc $)$ & $g_{/ /}(\exp )$ & $g_{/ /}($calc $) \Delta \& / g(\exp )$ \\
\hline Nd & $0.71(1)$ & 1.07 & $2.65(1)$ & 2.79 & $0.582(1)$ \\
Dy & $9.4(5)$ & 9.9 & $1.8(1)$ & 1.4 & $-0.678(6)$ \\
Er & $3.6(1)$ & 4.3 & $11.8(4)$ & 11.1 & $0.532(5)$
\end{tabular}

Table 3: Experimental $g$-factor and calculated ones for the $\mathrm{Ln}$ (trensal) series using the CFP reported in Table S4. The EPR investigation of the pure $\mathrm{Nd}$ (trensal) complex was conducted with a X-band spectrometer at $5 \mathrm{~K}$, while the values of the $g_{\text {erif }}$ for $\operatorname{Er}$ (trensal) and Dy (trensal) originate from a previous EPR W-band experiment on Y(III) diluted samples (3.1\% and $5.8 \%$ of Dy(III) and $\mathrm{Er}(\mathrm{III})$ respectively). ${ }^{54}$
Table 3).

If we take as a measure of the experimental anisotropy of the ground doublet the ratio $\left(g_{/ /}-g_{\perp}\right) /\left(g_{/ /}+g_{\perp}\right)$ it is easy to note that $\operatorname{Ln}($ trenovan) complexes are slightly more anisotropic than $\operatorname{Ln}($ trensal) ones; the difference which we essentially attribute to the shorter Ln-N apical distance - is however quite small, so that it cannot be consistently reproduced by the calculated CFPs. Further, the behavior of the magnetic anisotropy in the two series is consistent with the aspherical electron density distributions of the lanthanide ions, pioneered by Sievers ${ }^{55}$ and recently made popular by Rinehart and Long. ${ }^{4}$ The anisotropies of Ce and Dy derivatives have easy plane anisotropy while $\mathrm{Er}$ and $\mathrm{Yb}$ derivatives are easy axis. However, since both CFP analysis and EPR investigation are consistent with all the complexes being characterized by highly mixed ground states, deviation may occur. This is indeed the case of the $\mathrm{Nd}$ analogue, for which a weak easy axis anisotropy is observed, whereas a weak easy plane one could be expected on the basis of the Long model. This stresses once more that, even in relatively high symmetry system, the application of a model conceived for pure ground state should be considered with much caution.

non-Kramers ions. The EPR investigation of Lanthanide based complexes with integer spin is less straightforward than for Kramers ions, because it is common not to detect a signal, since the resonance condition is more difficult to be fulfilled. Even when a signal is observed, the more complex energy structure makes the spectrum interpretation not easy. ${ }^{56,57}$ The $\operatorname{Ln}($ trenovan) complexes with integer spin do not make an exception: we were able to identify a clear signal in the EPR spectrum of Tm(trenovan) and $\mathrm{Tb}$ (trenovan) only, while the spectra of $\operatorname{Pr}$ (trenovan) and Ho(trenovan) could not be safely assigned. However, as we will see below, caution should be exerted in the assignment of these signals to $\operatorname{Ln}($ trenovan). the high frequency EPR spectra of Tm(trenovan) were characterized by a single resonance, the magnetic field of which is varying linearly as a function of microwave frequency (see Figure S11). On the assumption that the observed transitions are parallel ones we can interpret the EPR spectra according to an effective doublet Hamiltonian:

$$
H=g_{/ /} B \boldsymbol{S}_{z}+\Delta \boldsymbol{S}_{x}+\Delta \boldsymbol{S}_{y}
$$


Where $\Delta$ is the energy gap in zero field between the two levels for which the transition is observed ${ }^{8}$ and the $\boldsymbol{S}_{x}$ and $\boldsymbol{S}_{y}$ terms arise as a consequence of the transverse terms of the CF. The resonance field vs frequency plot then allows to get a lower limit estimate of this energy gap $\Delta$, which is obtained as $9 \mathrm{~cm}^{-1}$, in reasonable agreement with analysis of CFPs. However, the linear dependence of the resonance field, with an estimated effective $g$ value of 8.9 , is completely at odds with the results of magnetic characterization, which indicated two low lying singlets with extremely small effective $g$ values. Further, one has to consider that to get a reasonable transition probability, either the polarization of the microwave should be parallel to the applied field, or the local symmetry at low temperature should be lower than trigonal, allowing for further mixing of different $M_{j}$ states. $^{8}$

A similar situation occurs for $\mathrm{Tb}$ (trenovan), which suggests a roughly linear field/frequency dependence: in this case, the measurements in X-band evidenced a transition in zero field with four lines, that are attributable to the hyperfine interactions with a $I=3 / 2$ nucleus (see Figure S11) while the analysis of high frequency data suggests an energy gap of $2 \mathrm{~cm}^{-1}$. None of these results are compatible with a ground singlet in a trigonal symmetry, so that we suggest that, in analogy to what observed for $\mathrm{Tb}$ (trensal), ${ }^{12}$ the observed spectra for Tm(trenovan) and $\mathrm{Tb}$ (trenovan) are actually due to an impurity characterized by different CFPs..$^{*}$

Y:Gd(trenovan). The anisotropy of the Gd(trenovan) complex was investigated by measuring the $\mathrm{W}$-band spectrum of the corresponding isostructural and diamagnetic doped Y-phase to obtain a better resolution of the spectrum. The spectrum was interpreted on the basis of the following spin Hamiltonian in Stevens' notation:

$$
\boldsymbol{H}_{\mathrm{SH}}=b_{2}^{0} \boldsymbol{O}_{2}^{0}+b_{44}^{0} \boldsymbol{O}_{4}^{0}+\mu_{\mathrm{B}} \boldsymbol{S} \cdot \boldsymbol{g} \cdot \boldsymbol{B}
$$

With $S=7 / 2$ and $b_{2}^{0}=D / 3$, where $D$ is the commonly employed axial zero-field splitting parameter.

The best simulation of the EPR spectrum gave $D=$ $(0.0615 \pm 0.0005) \mathrm{cm}^{-1}, b_{4}^{0}=4.5 \times 10^{-5} \mathrm{~cm}^{-1}$ and $\mathrm{g}=$ 1.995. The values of the $D$ and $g$ parameters are consistent with those of other Gd(III) -based complexes: ${ }^{58,59}$ these complexes are indeed quite isotropic since Gd(III) has the $4 f$ shell half-filled and the orbital contribution to the angular momentum cancels so that spin-orbit coupling only acts as a second order perturbation to introduce anisotropy. A few words should be spent on the determination of the sign of the $D$ parameter. It is well established that the determination of the sign of $D$ would require to study the variation of the EPR spectrum as a function of temperature, in order to observe a change in the intensity of the outermost transitions, either parallel or perpendicular. $^{60}$ However, this was not feasible for $\mathrm{Y}: \mathrm{Gd}($ trenovan) because only the spectrum collected at $10 \mathrm{~K}$ had a signal to noise ratio good enough to evidence clearly these transitions, which were further of quite weak intensity. Nevertheless, the analysis of the relative intensity of adjacent parallel transitions, simulated by using either $D=0.0615$ or $D=-0.0615$ and fourth-order term of corresponding sign, strongly supported the choice of $D>$ 0 (see also Figure 4). We note here that this is a relevant advantage of the use of W-band spectrometer compared to the X-band one: indeed, the spectrum could well be observed even at lower field/frequency, but due to the small zero field splitting all the eight levels would have been equipopulated at attainable laboratory temperatures, thus hampering the sign determination. Finally, we note that the spectra were not resolved enough to obtain reasonable estimates of trigonal anisotropy parameters $\left(b_{4}^{3}\right.$,

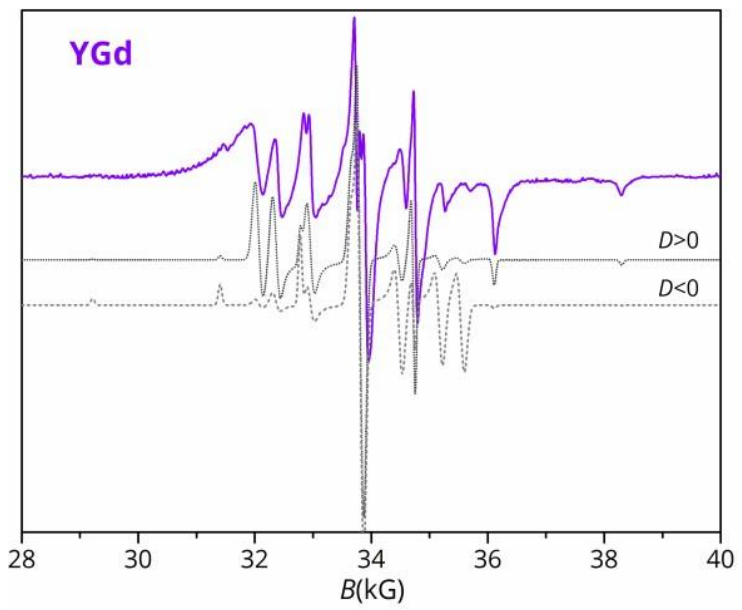

Figure 4: W-band EPR spectrum (violet solid line) for YGd(trenovan), along with its simulation (dotted and dashed lines) obtained with Easyspin. See text for the values of the simulation parameters.

$b_{6}^{3}$ and $b_{6}^{6}$ ) which were then not included in the simulation Hamiltonian. With the obtained parameters, the system can be considered to have extremely weak easy plane anisotropy $(D>0)$, and a global energy splitting of the ground multiplet is around $1 \mathrm{~K}\left(D\left(S^{2}-1 / 4\right)\right)$. 
Magnetic relaxation. The investigation of the magnetization dynamics for the $\operatorname{Ln}$ (trenovan) series evidenced that only compounds that contains Kramers' ions exhibit a non-zero imaginary susceptibility and only in presence of an applied magnetic field. The absence of slow magnetic relaxation for non-Kramers ions in $\operatorname{Ln}$ (trenovan) is in agreement with the electronic structure derived in the previous sections: all of them are characterized by a

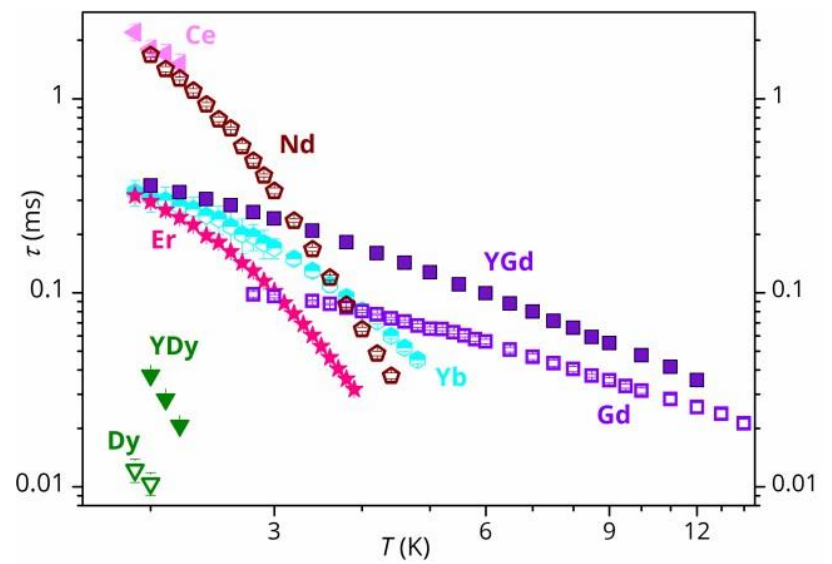

Figure 5: Temperature dependence of the magnetic relaxation time for the samples of the $\operatorname{Ln}($ trenovan) family.

ground singlet, for which slow relaxation is not expected. Moreover, the magnetic relaxation is clearly not related to the magnetic anisotropy of the complexes, since both compounds with easy axis and easy plane anisotropy showed a non-zero out of phase susceptibility. An analogue behavior had been first reported for $\operatorname{Er}$ (trensal) and Dy (trensal) $)^{54}$ and is now quite commonly reported, being attributed to relaxation processes different from the Orbach ones.

The magnetic relaxation was firstly studied as a function of the applied magnetic field at $2 \mathrm{~K}$ : the field which provided the maximum of the imaginary susceptibility, was then chosen as optimum field and used to perform the measurements as a function of temperature (see Figures S12-S17). It is worth noting that the scan in field at $2 \mathrm{~K}$ also evidenced a secondary maximum in the imaginary susceptibility for applied fields greater than 2000 Oe: its intensity however decreases very rapidly with temperature and it is no longer visible above $5 \mathrm{~K}$ (Figure S18). Moreover, this peak is completely absent in the diluted samples YGd(trenovan) and YDy(trenovan) (see Figures S19S21). This behavior suggests that the presence of this secondary peak in $\chi$ " may be due to dipolar interactions between different paramagnetic centers. Indeed, a similar feature was observed also in pure $\operatorname{Er}$ (trensal) and Dy(trensal) samples.

The temperature dependence of the relaxation time for the compounds of the series is reported in Figure 5. Although the $\mathrm{Sm}$ (trenovan) complex also showed a weak non-zero imaginary susceptibility, we could not exclude that the observation of a slow dynamics might be traced back to the Gd impurity. This stresses once more the importance of an integrated approach to the characterization of slow relaxing lanthanide complexes, where AC susceptibility results are complemented by spectroscopic outcomes.

A quantitative study of the mechanisms involved in the magnetic relaxation was not feasible, since it would have required to investigate the diluted analogues of the samples, which were available only for Dy and Gd. However, it is evident from the $\log$ - $\log$ plot in Figure 5 that the relaxation time of the investigated pure complexes presents two types of regimes that can be attributable to a Ramanlike mechanism (at higher temperatures) and a direct process (at lower temperature).

The different observed behavior between pure and diluted systems for Gd and Dy derivatives clearly the dynamic properties in the other systems are not purely molecular in nature, and are affected by the spin concentration in the lattice. It is then not to be attributed simply to an Orbach process as is also evident by the absence of linearity in the corresponding Arrhenius plots (see Figure S22).

As regards $\mathrm{YDy}$ (trenovan), above $2.2 \mathrm{~K}$ its dynamics was too fast to be measured with our AC susceptometer: indeed, the corresponding relaxation time, extracted by fitting the $\chi$ " curves with the Casimir Dupré equation, ${ }^{61}$ is available only for three temperatures and it is not thus suitable for discussion (see Figure 5). In the case of YGd(trenovan), it is possible to confirm that the relaxation is not promoted by an Orbach process, since EPR results showed that the whole $S=7 / 2$ multiplet spans only $1 \mathrm{~K}$ and $D$ value is positive. Indeed, a simple check of the YGd(trenovan) relaxation time above $7 \mathrm{~K}$ by means of the Arrhenius law would give an energy barrier of $10 \mathrm{~cm}^{-1}$, that, in this case, has no physical meaning (see Figure S23).

\section{CONCLUSIONS}

We have prepared and characterized a new family of lanthanide complexes in which most of the Kramers' ions 
derivatives behave as single-ion magnets ( $\mathrm{Ce}, \mathrm{Nd}, \mathrm{Gd}$, $\mathrm{Dy}, \mathrm{Er}$ and $\mathrm{Yb}$ ). For the study of their magnetic properties we have designed a strategy based on the structurally and chemically related $\operatorname{Ln}$ (trensal) family, which has been studied deeply in the literature. We have systematically completed these previous studies by combining spectroscopic and magnetic data to perform a detailed theoretical analysis using the CONDON package. These results have been an excellent starting point to reproduce the magnetic data and discuss the outcome of the EPR experiments.

As a whole, these results indicate once more that this type of complexes does not behave as 'real' SMM, if we denote with this term systems for which relaxation can be engineered by varying the anisotropy. This is clearly related to the charge distribution of the ligand around the lanthanoid being not clearly axial nor equatorial, which results in highly mixed ground states in terms of $M_{f}$ composition. This was also nicely confirmed by the results of EPR spectroscopy on Kramers derivatives, showing that passing from trensal to trenovan ligands, while keeping the same point symmetry at the lanthanides, slightly increases the ligand field strength along the series. The crucial parameter in determining the slow relaxation of the magnetization is then rather the number of unpaired electrons, only Kramers' ions showing in-field slow relaxation.

Finally, this study evidences that the analysis of a complete series such as that of $\operatorname{Ln}$ (trenovan) can be of help in elucidating trends in electronic and magnetic properties of Ln based complexes. In this respect it is to point out that the electronic structures obtained for Kramers ions of the Ln(trenovan) series follow a much reasonable trend, thanks to the observation of clear EPR spectra which imposed relevant constraints on CFPs values; on the other hand, the situation is less clear-cut for those system for which no EPR spectra could be safely assigned, such as Tm and Ho derivatives.

\section{ASSOCIATED CONTENT}

Supporting Information. The Supporting Information is available free of charge on the ACS Publications website.

\section{Present Addresses}

† Department of Chemistry "U.Schiff", C.R.I.S.T, Università degli Studi di Firenze, Via della Lastruccia 3-13, 50019 Sesto Fiorentino (FI), Italy.

\section{Author Contributions}

The manuscript was written through contributions of all authors. All authors have given approval to the final version of the manuscript. $\neq$ These authors contributed equally.

\section{Funding Sources}

No competing financial interests have been declared.

\section{Notes}

$1^{*}$ We note here that the high resolution powder X-ray diffractogram of $\mathrm{Tm}$ (trenovan) shows some weak extra peak compared to the calculated one, pointing to the presence of an unidentified phase with abundance $<5 \% \%$ (see Figure S1). A similar situation occurs for $\mathrm{Tb}$ (trenovan). Despite the low abundance of the spurious phase in the two samples, these might well be held responsible for the EPR signal observed, which is extremely sensitive to the presence of even a small abundance of paramagnetic centers.

\section{ACKNOWLEDGEMENT}

E. L. and L. S. acknowledge the financial support of MIUR through the project Futuro in Ricerca 2012 (RBFR12RPD1). Dr. G. Giambastiani and Dr. G. Tucci (ICCOM-CNR Florence) are gratefully acknowledged for TGA measurements and Dr. J. van Leusen for sharing his knowledge of the CONDON package and useful discussions. J. J. B. acknowledges support from the EU (ERC-2016-AdG-694097 QSpec-New Mat and COST Actions CA15128 Molecular Spintronics (MOLSPIN)) and the Basque Government "Grupos Consolidados UPV/EHU" (IT578-13)".

\section{ABBREVIATIONS}

XRPD, X-ray powder diffraction; CF, Crystal Field; EPR, electron paramagnetic resonance;

\section{AUTHOR INFORMATION}

\section{Corresponding Authors:}

${ }^{*}$ E-mail: josejaime.baldovi@ehu.eus

*E-mail: lorenzo.sorace@unifi.it 


\section{REFERENCES}

(1) Ishikawa, N.; Sugita, M.; Ishikawa, T.; Koshihara, S. Y.; Kaizu, Y. Lanthanide Double-Decker Complexes Functioning as Magnets at the Single-Molecular Level. J. Am. Chem. Soc. 2003, 125 (29), 8694-8695 DOI: $10.1021 / \mathrm{ja} 029629$ n.

(2) Woodruff, D. N.; Winpenny, R. E. P.; Layfield, R. A. Lanthanide Single-Molecule Magnets. Chem. Rev. 2013, 113 (7), 5110-5148 DOI: 10.1021/cr400018q.

(3) Sorace, L.; Gatteschi, D. Electronic Structure and Magnetie Properties of Lanthanide Molecular Complexes. In Lanthanides and Actinides in Molecular Magnetism; Layfield, R., Murugesu, M., Eds.; John Wiley \& Sons, 2015.

(4) Rinehart, J. D.; Long, J. R. Exploiting Single-Ion Anisotropy in the Design of F-Element Single-Molecule Magnets. Chem. Sci. 2011, 2 (11), 2078-2085 DOI: 10.1039/clsc00513h.

(5) Baldovi, J. J.; Cardona-Serra, S.; Clemente-Juan, J. M.; Coronado, E.; Gaita-Arifio, A.; Palii, A. Rational Design of Single-Ion Magnets and Spin Qubits Based on Mononuclear Lanthanoid Complexes. Inorg. Chem. 2012, 51 (22), 12565-12574 DOI: 10.1021/ic302068c.

(6) Vincent, R.; Klyatskaya, S.; Ruben, M.; Wernsdorfer, W.; Balestro, F. Electronic Read-out of a Single Nuclear Spin Using a Molecular Spin Transistor. Nature 2012, 488 (7411), 357-360 DOI: 10.1038/naturel1341.

(7) Rinehart, J.; Long, J. Exploiting Single-Ion Anisotropy in the Design of F-Element Single-Molecule Magnets. Chem. Sci. 2011, 2, 2078 DOI: 10.1039/clsc00513h.

(8) Abragam, A.; Bleaney, B. Electron Paramagnetic Resonance of Transition Ions; Oxford University Press, 1970.

(9) Liddle, S. T.; van Slageren,J. Improving F-Element Single Molecule Magnets. Chem. Soc. Rev. 2015, 44 (19), 6655-6669 DOI: 10.1039/ c5cs00222b.

(10) Magnani, N.; Caciuffo, R.; Colineau, E.; Wastin, F.; Baraldi, A.; Buffagni, E.; Capelletti, R.; Carretta, S.; Mazzera, M.; Adroja, D. T.; Watanabe, M.; Nakamura, A. Low-Energy Spectrum of a Tm-Based DoubleDecker Complex. Phys. Rev. B 2009, 79 (10), 104407 DOI: 10.1103/PhysRevB.79.104407.

(11) Flanagan, B. M.; Bernhardt, P. V.; Krausz, E. R.; Liithi, S. R.; Riley, M. J. A Ligand-Field Analysis of the Trensal (H3trensal = 2,2',2' '-Tris(salicylideneimino)triethylamine) Ligand) Ligand. An Application of the Angular Overlap Model to Lanthanides. Inorg. Chem. 2002, 41 (20), 5024-5033 DOI: 10.1021/ic011276q. 
(12) Perfetti, M.j Lucaccini, E.j Sorace, L.i Costes,J.-P.j Sessoli, R. Determination of Magnetic Anisotropy in the Lntrensal Complexes ( $\mathrm{Ln}=\mathrm{Tb}, \mathrm{Dy}, \mathrm{Er})$ by Torque Magnetometry. Inorg. Chem. 2015, 54 (7), 30903092 DOI: $10.1021 /$ acs.inorgchem.5b00288.

(13) Bethe, H. A. Termaufspaltung in Kristallen. Ann. Phys. 1929, 395, 133-208.

(14) Urland, W. On the Ligand-Field Potential for F Electrons in the Angular Overlap Model. Chem. Phys. 1976, 14 (3), 393-401 DOI: 10.1016/0301-0104(76)80136-X.

(15) Malta, O. L. A Simple Overlap Model in Lanthanide Crystal-Field Theory. Chem. Phys. Lett. 1982, 87 (1), 27-29 DOI: 10.1016/0009-2614(82)83546-X.

(16) Porcher, P.j Couto Dos Santos, M.j Malta, O. Relationship between Phenomenological Crystal Field Parameters and the Crystal Structure: The Simple Overlap Model. Phys. Chem. Chem. Phys. 1999, 1 (3)i 397-405 DOI: 10.1039/a803807d.

(17) Baldovi, J. J.i Borras-Almenar, J. J.i Clemente-Juan, J. M.j Coronado, E.i Gaita-Arifio, A. Modeling the Properties of Lanthanoid Single-Ion Magnets Using an Effective Point-Charge Approach. Dalt. Trans. 2012, 41 (44), 13705 DOI: 10.1039/c2dt31411h.

(18) Chibotaru, L. F.j Ungur, L. Ab Initio Calculation of Anisotropie Magnetic Properties of Complexes. I. Unique Definition of Pseudospin Hamiltonians and Their Derivation. J. Chem. Phys. 2012, 137 (6) DOI: 10.1063/1.4739763.

(19) Pedersen, K S.j Ungur, L.i Sigrist, M.j Sundt, A.i Schau-Magnussen, M.j Vieru, V.j Mutka, H.j Rols, S.j Weihe, H.i Waldmann, O.i Chibotaru, L. F.j Bendix,J.i Dreiser,J. Modifying the Properties of 4f Singlelon Magnets by Peripheral Ligand Functionalisation. Chem. Sci. 2014, 5 (4), 1650-1660 DOI: $10.1039 / \mathrm{c} 3 \mathrm{sc} 53044 \mathrm{~b}$.

(20) Aravena, D.i Atanasov, M.j Neese, F. Periodic Trends in Lanthanide Compounds through the Eyes of Multireference $\mathrm{Ab}$ Initio Theory. Inorg. Chem. 2016, 55 (9), 4457-4469 DOI: 1O.I021/ acs.inorgchem.6b00244.

(21) Baldovi, J. J.i Duan, Y.j Morales, R.j Gaita-Arifio, A.i Ruiz, E.j Coronado, E. Rational Design of Lanthanoid Single-Ion Magnets: Predictive Power of the Theoretical Models. Chem. Eur. J. 2016, 22 (38), 13532-13539 DOI: 10.1002/chem.201601741.

(22) Marx, R.j Moro, F.j Dorfel, M.j Ungur, L.i Waters, M.j Jiang, S. D.j Orlita, M.j Taylor, J.i Frey, W.j Chibotaru, L. F.j van Slageren, J. Spectroscopic Determination of Crystal Field Splittings in Lanthanide Double Deckers. Chem. Sci. 2014, 5 (8), 3287 DOI: 10.1039/c4sc00751d.

(23) Speldrich, M.j Schilder, H.j Lueken, H.j Kogerler, P. A Computational Framework for Magnetic Polyoxometalates and Molecular Spin Structrures: CONDON 2.0. Isr. J. Chem.2011, 51, 215-227 DOI: 10.1002/ijch.201100013.

(24) van Leusen, J.i Speldrich, M.j Schilder, H.i Kogerler, P. Comprehensive Insight into Molecular Magnetism via CONDON: Full vs. Effective Models. Coord. Chem. Rev. 2015, 289, 137-148 DOI: 10.1016/j.ccr.2014.10.011. 
(25) Liu, S.; Yang, L. W.; Rettig, S. J.; Orvig, C. Bulky Ortho 3-Methoxy Groups on N4O3 Amine Phenol Ligands Producing Six-Coordinate Bis(ligand)lanthanide Complex Cations $[\mathrm{Ln}(\mathrm{H} 3 \mathrm{~L}) 2] 3+(\mathrm{Ln}=\mathrm{Pr}$, Gd; H3L = tris(((2-Hydroxy-3-Methoxybenzyl)amino)ethyl)amine). Inorg. Chem. 1993, 32 (12), 2773-2778 DOI: 10.1021/ic00064a031.

(26) Costes, J.-P.; Dahan, F.; Nicodème, F. Structure-Based Description of a Step-by-Step Synthesis of Homo- and Heterodinuclear (4f, 4f') Lanthanide Complexes. Inorg. Chem. 2003, 42 (20), 6556-6563 DOI: $10.1021 / \mathrm{ic034564q.}$

(27) Liu, J.; Yuan, K.; Leng, J.-D.; Ungur, L.; Wernsdorfer, W.; Guo, F.; Chibotaru, L. F.; Tong, M. A SixCoordinate Ytterbium Complex Exhibiting Easy-Plane Anisotropy and Field-Induced Single-Ion Magnet Behavior. Inorg. Chem. 2012, 51 (15), 8538-8544 DOI: 10.1021/ic301115b.

(28) Boultif, A.; Louer, D. Powder Pattern Indexing with the Dichotomy Method. J. Appl. Crystallogr. 2004, 37 (5), 724-731 DOI: 10.1107/S0021889804014876.

(29) Altomare, A.; Cuocei, C.; Giacovazzo, C.; Moliterni, A.; Rizzi, R.; Corriera, N.; Falcicchio, A. EXPO2013: A Kit of Tools for Phasing Crystal Structures from Powder Data. J. Appl. Crystallogr. 2013, 46 (4), 1231-1235 DOI: 10.1107/S0021889813013113.

(30) BrukerAXS, Diffracp. T. TOPAS 4.2 User Manual,Bruker-AXS GmbH; 2008.

(31) Bernhardt, P. V; Flanagan, B. M.; Riley, M.J. Isomorphous Lanthanide Complexes of a Tripodal N4O3 Ligand.Aust. J. Chem. 2000, 53 (3), 229-231 DOI: 10.1071/CH99175.

(32) Bain, G. A.; Berry, J. F. Diamagnetic Corrections and Pascal's Constants. J. Chem. Educ. 2008, 85 (4), 532-536 DOI: 10.1021/ed085p532.

(33) Barra, A. L.; Hassan, A. K.; Janoschka, A.; Schmidt, C. L.; Schiinemann, V. Broad-Band Quasi-Optical HF-EPR Spectroscopy: Application to the Study of the Ferrous Iron Center from a Rubredoxin Mutant. Appl. Magn. Reson. 2006, 30 (3-4), 385-397 DOI: 10.1007/BF03166208.

(34) Stoll, S.; Schweiger, A. EasySpin, a Comprehensive Software Package for Spectral Simulation and Analysis in EPR. J. Magn. Reson. 2006, 178 (1), 42-55 DOI: 10.1016/j.jmr.2005.08.013.

(35) Seitz, M.; Oliver, A. G.; Raymond, K. N. The Lanthanide Contraction Revisited. J. Am. Chem. Soc.2007, 129 (36), 11153-11160 DOI: 10.1021/JA072750F.

(36) Kanesato, M.; Yokoyama, T.; Itabashi, O.; Suzuki, T. M.; Shiro, M. Synthesis and Structural Characterization of Praseodymium(III) and Neodymium(III) Complexes of Tripodal Tris[2(Salicylideneamino)ethyl]amine. Bull. Chem. Soc. Jpn. 1996, 69 (5), 1297-1302 DOI: 10.1246/bcsj.69.1297.

(37) Kanesato, M.; Yokoyama, T. Synthesis and Structural Characterization of Ln (III) Complexes ( $\mathrm{Ln}=\mathrm{Eu}$, $\mathrm{Gd}, \mathrm{Tb}, \mathrm{Er}, \mathrm{Tm}, \mathrm{Lu}$ ) of Tripodal Tris (2-(Salicylideneamino) Ethyl) Amine. Chem. Lett. 1999, No.2, 137-138 DOI: 10.1246/cl.1999.137.

(38) Kanesato, M.; Yokoyama, T. Crystal Structures of Dysprosium(III) and Holmium(III) Complexes of 
Tripodal Tris[2-(Salicylideneamino)ethyl]amine. Anal. Sci. 2000, 16 (3), 335-336 DOI: 10.2116/analsci.16.335.

(39) Kanesato, M,i Mizukami, S,i Houjou, H,i Tokuhisa, H,i Koyama, E, $i$ Nagawa, Y. Comparison of the Bond Lengths for the Lanthanide Complexes of Tripodal Heptadentate Ligands. J. Alloys Compd. 2004, 374 (1),307-310 DOI: 10.1016/j.jallcom.2003.11.096.

(40) Bernhardt, P. V.i Flanagan, B. M,i Riley, M. J. Completion of the Isomorphous Ln ( Trensal ) Series. Aust.J. Chem. 2001, 54, 229-232 DOI: 10.1071/CH01076.

(41) Mizukami, S,i Houjou, H,i Kanesato, M,i Hiratani, K. Adjustment of Twist Angles in Pseudo-Helical Lanthanide Complexes by the Size of Metal Ions. Chem. Eur. J. 2003, 9 (7), 1521-1528 DOI: 10.1002/chem.200390174.

(42) Kanesato, M, i Nagahara, K.i Igarashi, K.i Sato, K.i Kikkawa, Y.i Goto, M. Synthesis, Characterization and Emission Properties of yttrium(III) and europium(III) Complexes of a Tripodal Heptadentate SchiffBase Ligand $\mathrm{N}[\mathrm{CH} 2 \mathrm{CH} 2 \mathrm{NCH}(2-\mathrm{OH}-3-\mathrm{MeC} 6 \mathrm{H} 3)] 3$. Inorganica Chim. Acta 2011, 367 (1), 225-229 DOI: $10.1016 /$ j.ica.2010.12.001.

(43) Nagahara, Ki Kanesato, M, i Sato, Ki Midori, G. Synthesis and Crystal Structure of a Terbium(III) Complex of Tris(2-(2-Hydroxy-3-Methylbenzylideneamino)ethyl)amine. X-ray Struct. Anal. Online 2011, 27, 9-10 DOI: 10.2116/xraystruct.27.9.

(44) Gorller-Walrand, C.i Binnemans, K. Razionalization of Crystal Field Parametrization. In Handbook on the Physics and Chemistry of Rare Earthsi Gscneidner jr., K. -A., Eyring, L., Eds,i Elsevier, 1996i Vol. 23, pp 121-283.

(45) Wybourne, B. G. Spectroscopic Properties of Rare Earthsi]ohn Wiley and Sons, 1965.

(46) Lucaccini, E, $i$ Briganti, $M, i$ Perfetti, $M, i$ Vendier, L.i Costes, J.-P, i Totti, F, $i$ Sessoli, R, $i$ Sorace, L. Relaxation Dynamics and Magnetic Anisotropy in a Low-Symmetry Dym Complex. Chem. Eur. J. 2016, 22 (16), 5552-5562 DOI: 10.1002/chem.201505211.

(47) Baldovi, J. J.i Cardona-Serra, S,i Clemente-Juan,J. M, i Coronado, E, i Gaita-Arifio, A.i Palii, A. SIMPRE: A Software Package to Calculate Crystal Field Parameters, Energy Levels, and Magnetic Properties on Mononuclear Lanthanoid Complexes Based on Charge Distributions. J. Comput. Chem. 2013, 34 (22), 1961-1967 DOI: 10.1002/jcc.23341.

(48) Baldovi, J. J.i Clemente-Juan, J. M, i Coronado, E, i Gaita-Arifio, A.i Palii, A. An Updated Version of the Computational Package SIMPRE That Uses the Standard Conventions for Stevens Crystal Field Parameters.J. Comput. Chem.2014,35 (26), 1930-1934DOI: 10.1002/jcc.23699.

(49) Flanagan, B. M,i Bernhardt, P. V.i Krausz, E. R.i Liithi, S. R,i Riley, M. J. Ligand-Field Analysis of an Er(III) Complex with a Heptadentate Tripodal N4O3 Ligand. Inorg. Chem. 2001, 40 (21), 5401-5407 DOI: $10.1021 / \mathrm{ic} 0103244$.

(50) Rechkemmer, Y.i Fischer, J. E.i Marx, R.i Dorfel, M,i Neugebauer, P, i Horvath, S,i Gysler, M,i BrockNannestad, $T, i$ Frey, W,i Reid, M. F, $i$ and van Slageren,J. Comprehensive Spectroscopic Determination 
of the Crystal Field Splitting in an Erbium Single-Ion Magnet. J. Am. Chem. Soc. 2015, 137 (40), 1311413120 DOI: $10.1021 /$ jacs.5b08344.

(51) Pedersen, K. S,i Dreiser, J.i Weihe, H,i Sibille, R,i Johannesen, H. Vi SOrensen, M. A.i Nielsen, B. E,i Sigrist, M, $i$ Mutka, H.i Rols, S, $i$ Bendix,J.i Pilingkos, S. Design of Single-Molecule Magnets: Insufficiency of the Anisotropy Barrier as the Sole Criterion. Inorg. Chem. 2015, 54 (15), 7600-7606 DOI: 10.1021/acs.inorgchem.5b01209.

(52) Vonci, $M, i$ Giansiracusa, $M$,J.i Van den Heuvel, $W, i$ Gable, R. W, $i$ Moubaraki, B. $i$ Murray, K. S, $i$ Yu, D, $i$ Mole, R. A.i Soncini, A.i Boskovic, C. Magnetic Excitations in Polyoxotungstate-Supported Lanthanoid Single-Molecule Magnets: An Inelastic Neutron Scattering and Ab Initio Study. Inorg. Chem. 2017, 56 (1), 378-394 DOI: 10.1021/acs.inorgchem.6b02312.

(53) Lucaccini, E. Master Thesis, University ofFlorence, 2013.

(54) Lucaccini, E, $i$ Sorace, L. $i$ Perfetti, $M, i$ Costes, J.-P. J.-P, $i$ Sessoli, R. Beyond the Anisotropy Barrier: Slow Relaxation of the Magnetization in Both Easy-Axis and Easy-Plane Ln (Trensal) Complexes. Chem. Commun. 2014, 50 (14), 1648-1651 DOI: 10.1039/c3cc48866g.

(55) Sievers, J. Asphericity of 4f-Shells in Their Hund's Rule Ground States. Zeitschrift fur Phys. B Condens. Matter 1982, 45 (4), 289-296 DOI: 10.1007/BF01321865.

(56) Ghosh, S,i Datta, S,i Friend, Li Cardona-Serra, S,i Gaita-Arifio, A.i Coronado, E,i Hill, S. MultiFrequency EPR Studies of a Mononuclear Holmium Single-Molecule Magnet Based on the Polyoxometalate [HoIII(W5O18)2]9-. Dalt. Trans. 2012, 41 ( 44), 13697 DOI: 10.1039/c2dt31674a.

(57) Baker, J. M,i Hutchison, C. A.i Leask, M. J. M,i Martineau, P. M,i Robinson, M. G. Electron Paramagnetic Resonance, Optical and Magnetic Studies of Tb(III) in Lanthanide Nicotinate Dihydrates. Proc. $R$ Soc. LondonAMath. Phys. Eng. Sci. 1987, 413 (1845) DOI: 10.1098/rspa.1987.0129.

(58) Martinez-Pérez, M. J.i Cardona-Serra, S,i Schlegel, C.i Moro, F,i Alonso, P. J.i Prima-Garda, H,i Clemente-Juan, J. M, i Evangelisti, M,i Gaita-Arino, A.i Sesé, J.i Van Slageren, J.i Coronado, E, $i$ Luis, F, $i$ Gaita-Arifio, A.i Sesé, J.i others. Gd-Based Single-Ion Magnets with Tunable Magnetic Anisotropy: Molecular Design of Spin Qubits. Phys. Rev. Lett. 2012, 108 (24), 247213 DOI: 10.1103/PhysRevLett.108.247213.

(59) Caneschi, A.i Dei, A.i Gatteschi, D, i Massa, C. A.i Pardi, L. A.i Poussereau, S, i Sorace, L. Evaluating the Magnetic Anisotropy in Molecular Rare Earth Compounds. Gadolinium Derivatives with Semiquinone Radical and Diamagnetic Analogues. Chem. Phys. Lett. 2003, 371 (5), 694-699 DOI: 10.1016/S00092614(03)00346-4.

(60) Barra, A.-L.i Brunel, L.-C.i Gatteschi, D,i Pardi, L.i Sessoli, R. High-Frequency EPR Spectroscopy of Large Metal Ion Clusters: From Zero Field Splitting to Quantum Tunneling of the Magnetization. Ace. Chem. Res. 1998, 31 (8), 460-466 DOI: 10.1021/AR960157P.

(61) Casimir, H. B. G,i Du Pré, F. K. Note on the Thermodynamic Interpretation of Paramagnetic Relaxation Phenomena. Physica 1938, 5,507-511 DOI: 10.1016/S0031-8914(38)80164-6. 
SYNOPSIS TOC (Word Style "SN_Synopsis_TOC").

A new series of trigonal lanthanide complexes, in which the Kramers derivatives Ce, Nd, Gd, Dy, Er and Yb show field induced slow relaxation of the magnetization is reported. Their electronic structures and magnetic anisotropies are elucidated using multifrequency EPR spectroscopy and magnetic susceptibility measurements, complemented by theoretical analysis using the CONDON package.
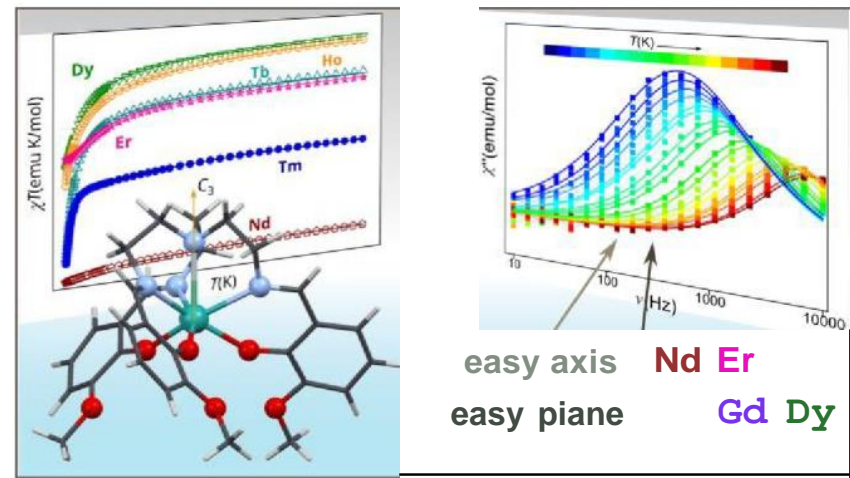